\title{
Temperature-dependence of the QCD topological susceptibility
}

\author{
Tamas G. Kovacs ${ }^{1, \star}$ \\ ${ }^{1}$ Institute for Nuclear Research of the Hungarian Academy of Sciences, H-4026 Debrecen, Hungary
}

\begin{abstract}
We recently obtained an estimate of the axion mass based on the hypothesis that axions make up most of the dark matter in the universe. A key ingredient for this calculation was the temperature-dependence of the topological susceptibility of full QCD. Here we summarize the calculation of the susceptibility in a range of temperatures from well below the finite temperature cross-over to around $2 \mathrm{GeV}$. The two main difficulties of the calculation are the unexpectedly slow convergence of the susceptibility to its continuum limit and the poor sampling of nonzero topological sectors at high temperature. We discuss how these problems can be solved by two new techniques, the first one with reweighting using the quark zero modes and the second one with the integration method.
\end{abstract}

\section{Introduction}

One of the most viable candidates for dark matter is the axion [1]. However, experimental search for this so far hypothetical particle is seriously hampered by the lack of information regarding its mass. The axion would also solve the long-standing strong CP problem of QCD due to its coupling to the QCD topological charge. A crucial ingredient for estimating the axion mass is the temperaturedependence of the QCD topological susceptibility, a quantity that proved to be notoriously hard to compute.

There are two main difficulties involved in the computation of the topological susceptibility at high temperature. Firstly, there are unexpectedly large cut-off effects that become worse as the temperature goes up. The origin of this problem can be easily understood from the underlying physics. At high temperature typical instantons become small, as their size is controlled by $1 / T$, the temporal extent of the system. Small instantons are badly resolved by a non-chiral Dirac operator and the would-be topological zero modes are far away from zero. As a result, the quark determinant cannot provide the appropriate suppression of higher topological sectors. As we will see, in a simulation with conventional methods, infeasibly fine lattices would be needed to resolve small instantons.

The second difficulty of the computation stems from the fact that above the finite temperature cross-over to the quark-gluon plasma, the topological susceptibility falls sharply with increasing temperature. The resulting tiny susceptibility is hard to measure since even in large volumes the system rarely visits non-trivial topological sectors. We emphasize that this is a physical property of the system and has nothing to do with the autocorrelation time of the used algorithm. However, there is

\footnotetext{
^Speaker, e-mail: kgt@atomki.mta.hu. Acknowledges financial support under contract number OTKA-K-113034. Thanks Z. Fodor, S.D. Katz and K.K. Szabó for numerous helpful discussions during the preparation of this talk. Most of the computations presented here were performed on JUQUEEN at Forschungszentrum Jülich.
} 
also a connected algorithmic problem that makes the situation even worse, and this is the slow topology change on fine lattices. These factors together make it prohibitively expensive to gather enough statistics for the susceptibility with current resources.

In the present work, based on Ref. [2], we compute the QCD topological susceptibility starting from the $T=0$ region through the transition temperature around $150 \mathrm{MeV}$ [3], up to $2 \mathrm{GeV}$, that is the whole temperature range relevant for axion cosmology. We offer solutions to both problems hampering the calculation of the susceptibility. We show that even without resorting to extremely fine lattices, the incorrect suppression of higher topological sectors can be cured by a suitable modification of the quark determinant. This involves shifting would-be zero modes to exactly zero and reweighting configurations with the ratio of the original determinant and the one with shifted zero modes.

The second problem, that of insufficient statistics for higher topological sectors, can be solved by noting that derivatives of the susceptibility (with respect to temperature and quark mass) are much easier to calculate than the susceptibility itself. Indeed, these derivatives can be written in terms of the average action and the quark condensates measured in different topological sectors. Using these derivatives, the susceptibility can be integrated up to any point in parameter space, starting from a point (low temperature, heavy quarks) where a direct calculation is feasible. This procedure results in the so called integral method that was independently also suggested for the quenched case by Frison et al. [4].

In the following, using reweighting and the integral method, we present a calculation of the QCD topological susceptibility in the temperature range $0<T<2 \mathrm{GeV}$. Our final results pertain to the real physical situation including the continuum limit and the effects of dynamical $u, d, s$ and $c$ quarks with their physical masses along with a correction for the $u-d$ isospin splitting.

\section{Unusually large cutoff effects}

For the simulations that we present here we used the tree-level improved Symanzik gauge action together with staggered fermions on four times stout-smeared gauge links [5]. The continuum limit was based on simulations with temporal lattice sizes of $N_{t}=8,10,12,16$ and 20. For the lowest temperature range, up to $T=250 \mathrm{MeV}$ our simulations include $2+1$ quark flavors with physical masses and for higher temperatures where it contributes significantly, we also included the charm quark. Some of our simulations were done with overlap quarks [6]. As explained later in the text, at intermediate stages some of our calculations involved simulations with heavier than physical $u, d$ quarks up to the strange quark mass $\left(N_{f}=3\right)$, but this was only needed for the integral method, no extrapolation in the quark mass was involved. For more details of our setup we refer the reader to $[2,7]$ and [8].

In this section we show why the topological susceptibility at high temperature suffers from unusually large cutoff effects. We will also describe how to solve this problem without utilizing extremely fine lattices that would make it prohibitively expensive to obtain the continuum limit with present-day resources.

\subsection{The problem}

To illustrate the cutoff effects we face here, in Fig. 1 we show the topological susceptibility at $T=$ $300 \mathrm{MeV}$, measured using conventional methods on lattices of different coarseness corresponding to $N_{t}=8,10,12$ and 16. This is a typical setup for taking the continuum limit in present-day QCD thermodynamics calculations. The data labeled "ratio" was obtained by normalizing the raw data with the zero temperature susceptibility at the same lattice spacing [9]. The first three points (from the 


\section{Topological susceptibility at $\mathrm{T}=300 \mathrm{MeV}$}

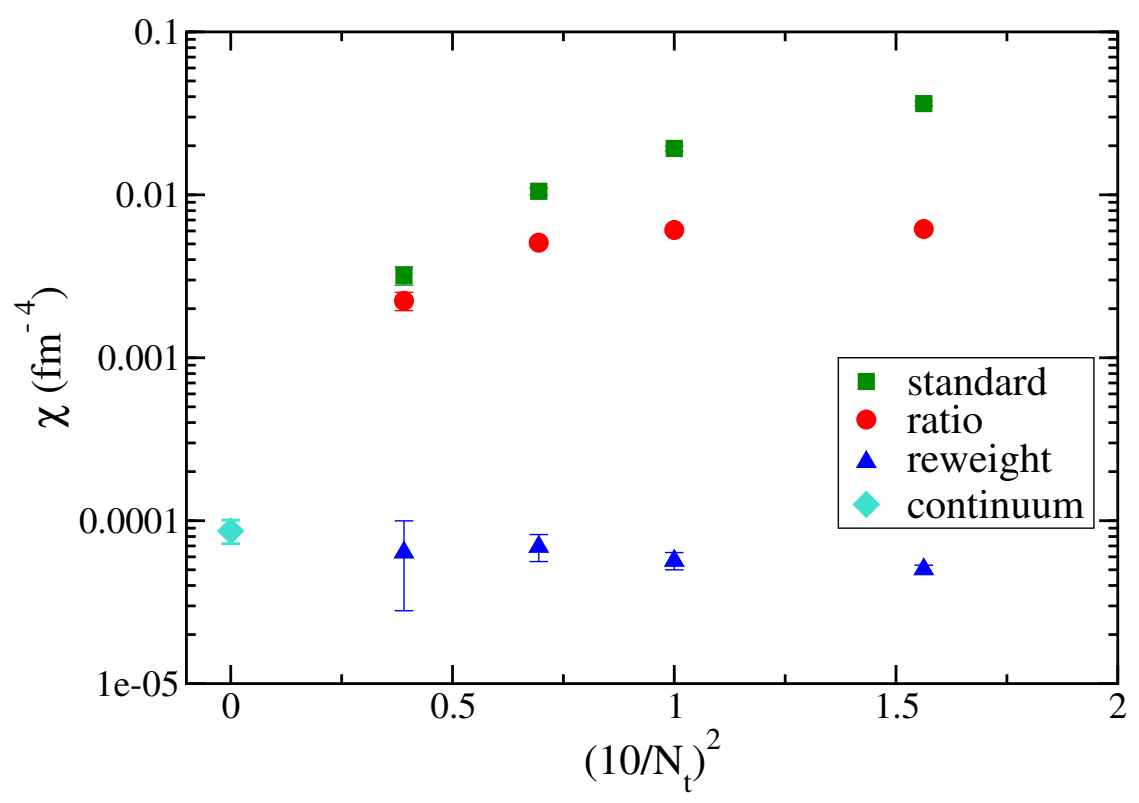

Figure 1. The dependence of the topological susceptibility on the lattice spacing at $T=300 \mathrm{MeV}$, obtained with two conventional methods (see text) and reweighting (to be discussed later). The horizontal axis is proportional to the lattice spacing squared. The continuum extrapolated point at zero was obtained using our reweighting method.

right) of this data set appear to be nicely scaling, but the point on the finest lattice reveals that scaling will probably set in only for much finer lattices. Indeed, for comparison we also included the correct continuum extrapolated value that our reweighting method (to be described) yields. Clearly, nothing comparable could have been obtained by extrapolating the standard or ratio data sets in the figure.

What is the the source of these unexpectedly large scaling violations for the susceptibility at high temperature? In particular why does one need much finer lattices at high temperature than at zero temperature for the continuum limit of the susceptibility? The answer to this question can be given by examining the typical size of instantons contributing to the susceptibility at different temperatures. At zero temperature the instanton size is limited by non-perturbative QCD physics and as a result typical instantons have a radius around 0.3-0.4 fm [10]. In contrast, above the cross-over to the quark-gluon plasma, the temporal size of the system becomes too small to accommodate these instantons. Therefore, at high temperature the typical instanton size is controlled by the temporal size of the system, $1 / T$ and the size of typical instantons dominating the susceptibility becomes smaller, proportionally to the inverse temperature.

In QCD with light dynamical quarks an important factor influencing the topological susceptibility is the suppression of higher topological sectors by the quark determinant. This suppression can mostly be attributed to the zero eigenvalues (zero modes) of the quark Dirac operator in the presence of topological charge. In the topological sector with charge $Q$ each quark flavor has (at least) $|Q|$ zero eigenvalues [11] contributing a factor $m_{f}^{|Q|}$ to the determinant, where $m_{f}$ is the mass of the given quark flavor. However, this is true only in the continuum limit. Topological zero modes of a non-chiral lattice 
Dirac operator do not occur at exactly zero. For a given lattice Dirac operator, how far an instanton zero mode is shifted away from zero depends mostly on the size of the corresponding instanton; the larger the instanton is in lattice units, the better it can be resolved by the lattice Dirac operator, the closer the would be zero mode is to zero.

Putting this all together we can understand the origin of large scaling violations. At high temperature typical instantons are small and the corresponding zero modes $\lambda_{0}$ are farther away from zero. As a result, the suppression factor they contribute in the quark determinant, $\lambda_{0}+m_{f}$ can be significantly different from the continuum factor $m_{f}$ and higher topological sectors are not sufficiently suppressed.

For a more quantitative understanding let us assume that we keep $N_{t}$, the temporal extent of the system in lattice units fixed and set the temperature by adjusting the gauge coupling $\beta$. Moving along the line of constant physics the quark mass in physical units remains constant (up to logarithmic corrections). However, the instanton zero modes $\lambda_{0}$ (again in physical units) will increase proportionally to the temperature. This is because the resolution of typical instantons do not change, their size remains roughly $N_{t}$ in lattice units and then the zero modes in lattice units $\lambda_{0} a$ also do not change which in turn implies that

$$
\lambda_{0} \propto \frac{1}{a} \propto T
$$

This means that in this setup at higher temperatures the zero mode factors $\lambda_{0}+m_{f}$ are more and more dominated by the increasing $\lambda_{0}$ and as a result, higher topological sectors are not properly suppressed. The foregoing discussion also suggests that if we wanted to compensate this effect by working on finer lattices, the lattice spacing would need to be made smaller proportionally to the inverse temperature. In the temperature range at hand this is clearly not feasible with presently available resources. It is also clear that if instead of increasing $\beta$, the temperature is raised by choosing smaller values of $N_{t}$, the resolution of typical instantons and, as a result, the scaling violations will become even worse.

\subsection{Reweighting}

Understanding the problem in this way also helps to find the solution. The appropriate suppression of higher topological sectors can be restored by shifting the would be zero modes to zero, where they would occur in the continuum limit.

As a first step we have to identify the would be zero modes produced by the topological charge. To this end we determine the topological charge of each configuration using the Wilson flow [12] and extracting the charge $Q$ from the smoothed gauge field. Next we determine the $4|Q|$ smallest magnitude eigenvalues of the staggered Dirac operator. We identify these as the would-be zero modes and shift them in the quark determinant to zero. This amounts to a reweighting of the given configuration with the factor

$$
w[U]=\prod_{f} \prod_{n=1}^{4|Q|}\left(\frac{m_{f}}{\lambda_{n}[U]+m_{f}}\right)^{1 / 4},
$$

where the product runs over the light quark flavors $(u, d, s)$. Notice that in a gauge field background with topological charge $Q$ the continuum Dirac operator would have $|Q|$ exact zero modes. To take into account the expected degeneracy of staggered quarks in the continuum limit, here we use the $4|Q|$ smallest magnitude modes for the reweighting, but, as usual in staggered simulations, also take the fourth root to restore the proper number of flavors in the determinant.

The reweighting is a non-local modification of the quark action, however, in the continuum limit the reweighting factors tend to unity and the original quark action is restored. This happens because on finer lattices the topological would be zero modes get closer to zero. We demonstrate this in Fig. 2 by plotting the distribution of the zero modes on lattices with different lattice spacings. The distribution 
can be seen to become narrower, typical lattice zero modes move closer to zero as the lattice becomes finer. As demonstrated in Fig. 3, we also explicitly checked that the reweighting factors tend to unity as the continuum limit is taken.

The reweighted susceptibility scales much better, as is apparent in Fig. 1. This indicates that the lack of scaling of the original non-reweighted data is indeed due to the failure of the Dirac operator to capture the zero modes properly and thereby failure to provide the proper suppression of higher topological sectors.

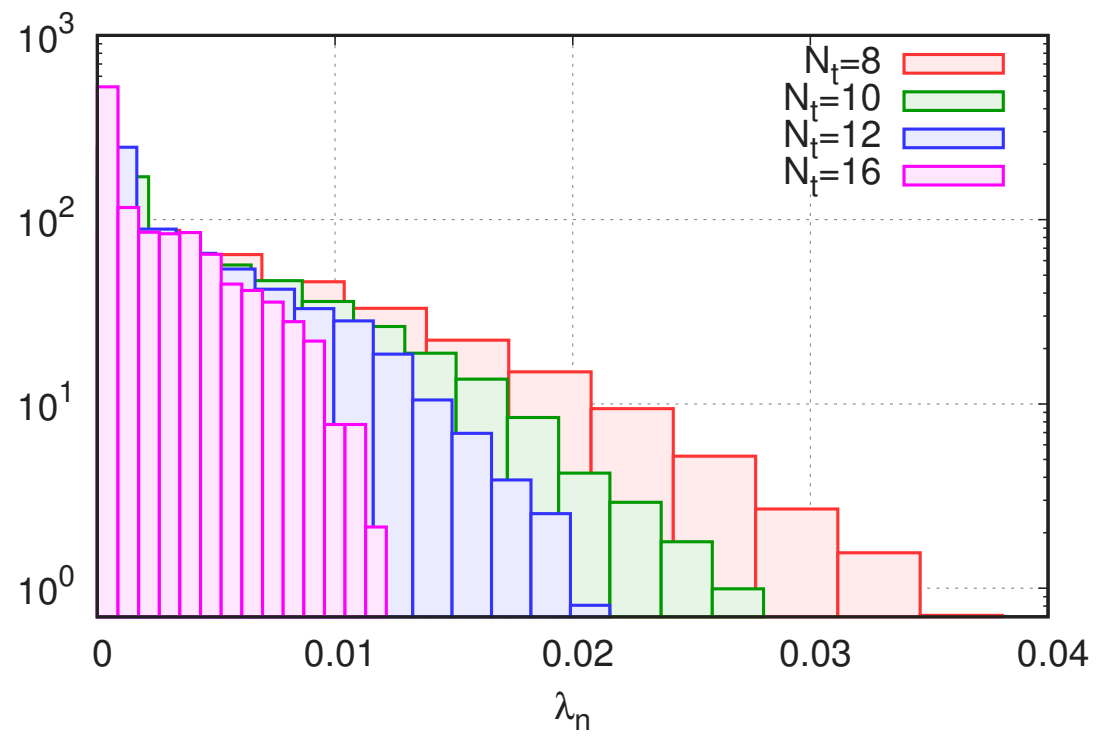

Figure 2. The distribution of the would-be zero modes of the staggered Dirac operator for different lattice spacings.

\section{The integral method}

As we already mentioned, the second challenge in computing the topological susceptibility at high temperature is the bad sampling of nonzero topological sectors. A quick estimate indicates that the direct calculation of the susceptibility up to $T=2 \mathrm{GeV}$ with reasonable accuracy would be approximately ten orders of magnitude beyond present-day computational resources.

Instead of a straightforward calculation of the susceptibility, here we propose to use the integral method. It is based on the simple fact that derivatives of the weights of different topological sectors are much easier to evaluate than the susceptibility itself. Indeed, a simple calculation reveals that

$$
\frac{d \log Z_{Q} / Z_{0}}{d \log T}=\frac{d \beta}{d \log a}\left\langle S_{g}\right\rangle_{Q-0}+\sum_{f} \frac{d \log m_{f}}{d \log a}\left\langle\bar{\psi}_{f} \psi_{f}\right\rangle_{Q-0},
$$

where $Z_{Q} / Z_{0}$ is the relative weight of topological sectors $Q$ and 0 and we introduced the notation

$$
\langle X\rangle_{Q-0}=\langle X\rangle_{Q}-\langle X\rangle_{0}
$$




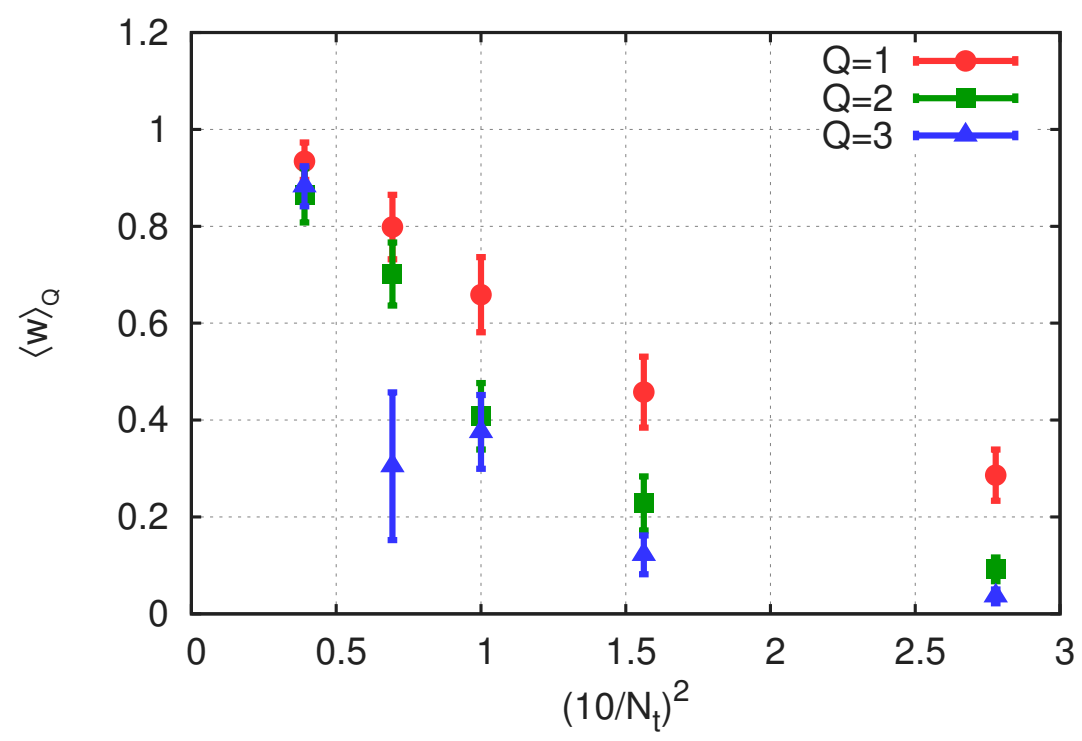

Figure 3. The average reweighting factors for different charge sectors as a function of the lattice spacing squared for the $N_{f}=3$ system at $T=300 \mathrm{MeV}$.

for the difference of expectations of the quantity $X$ in the topological sector $Q$ and zero. The right-hand side of Eq. 3 can be easily evaluated by measuring the gauge action $\left\langle S_{g}\right\rangle$ and the quark condensates $\left\langle\bar{\psi}_{f} \psi_{f}\right\rangle$ separately in the charge $Q$ and charge zero sectors via fixed topological sector simulations.

For the derivatives to be useful we also need the relative weight of different topological sectors at some fixed temperature $T_{0}$. We can choose $T_{0}$ to be low enough that a direct measurement of the weights is feasible. Starting from $T_{0}$ the weights can be integrated up to the desired higher temperature where they can be combined to provide the susceptibility.

Even further simplification occurs since at high temperature the instanton gas is so dilute that the weight of different sectors is precisely given by a free instanton gas. If this is the case, the probability distribution of the topological charge is already determined by $Z_{1} / Z_{0}$. Thus the susceptibility can also be written in terms of this quantity and the weight of higher sectors is not needed. In fact, already a bit above the cross-over a gas of free instantons provides a rather accurate description of the system. To show this, in Fig. 4 we compare the charge probability distribution obtained from our simulation with reweighting with that of a gas of free instantons. The density of the free gas was chosen to reproduce the topological susceptibility of the simulation data. We note that the system is at a fairly low temperature, just above the cross-over and it has a large spatial linear size $6.6 \mathrm{fm}$ potentially accommodating several instantons. In spite of that we do not see a significant difference between the simulation and the free gas.

Up to $T=300 \mathrm{MeV}$ direct simulations are also available and compatible with results obtained with the integral method. It is only above $300 \mathrm{MeV}$ that we resort to only the integral method and the assumption of free instantons. At that point the instanton gas is much more dilute than at the temperature shown in the figure so the free gas should provide the appropriate description. However, this only means that the interaction among instantons is neglected but the one-instanton weight $Z_{1} / Z_{0}$ 


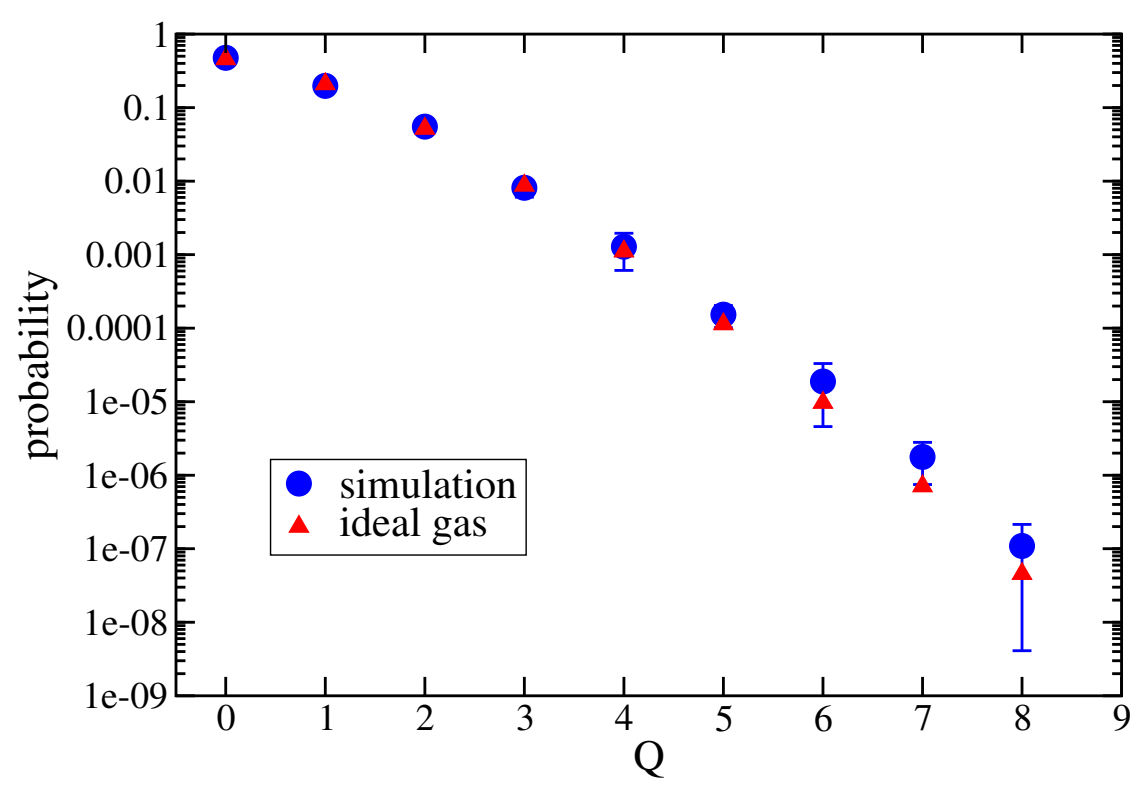

Figure 4. The probability of different topological sectors at $T=180 \mathrm{MeV}$ and $N_{t}=16$ in a system of spatial linear size $L=6.6 \mathrm{fm}$. A comparison is made between our simulation data obtained with reweighting and a gas of free instantons with its density adjusted to provide the same topological susceptibility.

in the partition function is still determined fully non-perturbatively. We emphasize that our approach is different from what is known in the literature as the DIGA (dilute instanton gas approximation) where the perturbative result is used for the one-instanton weight.

Additional savings in the computing resources can be had by noticing that integration in terms of the light quark masses is cheaper than integration in the temperature. Therefore at the reference point $T_{0}$ we can use a system with heavy $u$ and $d$ quarks that are chosen to be degenerate with the physical $s$ quark. Due to the heavier $u$ and $d$ quarks the temperature integration of the weights in this $N_{f}=3+1$ flavor system is much cheaper than it would be in the physical $N_{f}=2+1+1$ system. To obtain the susceptibility for physical quark masses, we can integrate the weights in terms of the $u$ and $d$ quark masses starting from the $N_{f}=3+1$ theory at the desired temperature. This integration in terms of the light quark masses was carried out using $N_{f}=2+1$ overlap simulations. In Fig. 5 we depict a schematic view of the simulations in the temperature versus $(u, d)$ quark mass plane. The arrows indicate the paths of integration for determining the weights of topological sectors. We also show in the figure the regions where different methods (reweighting without integration and the standard method without reweighting or integration) were used independently. Throughout the overlapping regions the different methods gave consistent results.

\section{Conclusions}

Finally, in Fig. 6 we summarize our main result, the temperature-dependence of the QCD topological susceptibility. In the same figure we compare our results to two other determinations of the susceptibility that appeared recently in the literature. Our results appear to be significantly lower than those of Bonati et al. [9]. However, the susceptibility obtained by Petreczky at al. [14] with one of their 


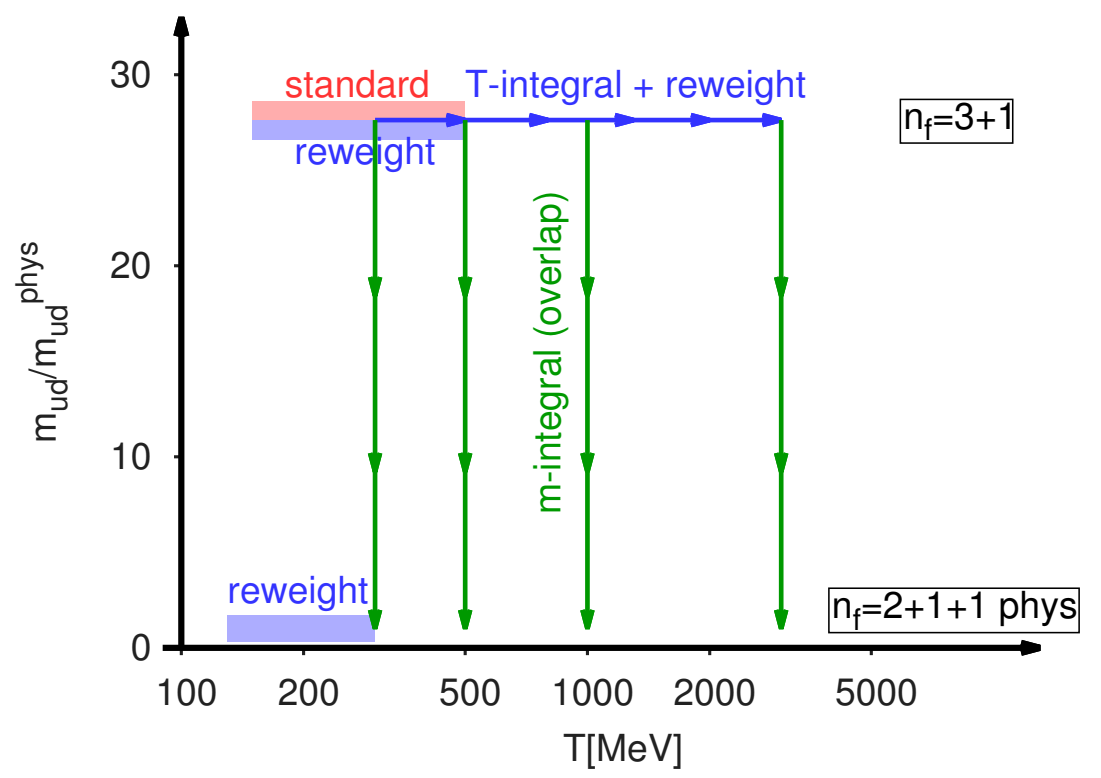

Figure 5. Schematic view of the simulations we have performed in the temperature versus light quark mass plane. The arrows indicate the paths of integration for the topological sector weights. We also marked the regions where only reweighting (no integration) and where the standard method (no reweighting, no integration) was used.

methods is consistent with ours, albeit our results have much smaller uncertainties compared to theirs. In addition, our results, going all the way up to $T=2 \mathrm{GeV}$, cover a much wider temperature range than the other results in the literature.

The important point we would like to emphasize is that the topological susceptibility at high temperature has much larger cutoff corrections than most other quantities. Lattices that are sufficiently fine for typical QCD thermodynamics are much too coarse to obtain a reliable continuum extrapolation of the topological susceptibility. We demonstrated that these unusually large lattice artifacts are due to small instantons and the inability of non-chiral Dirac operators to properly describe their zero modes. We showed that lattice zero modes occurring far away from zero and cannot provide enough suppression for higher topological sectors and the topological susceptibility can be grossly over-estimated. We also argued that this problem can be solved by shifting the lattice zero modes to zero where their continuum counterparts would occur and reweighting lattice configurations accordingly. An alternative possibility would be to use a chiral quark action, like the overlap where lattice zero modes are protected by an exact Ginsparg-Wilson symmetry.

\section{References}

[1] G.D. Moore, Axion dark matter and the Lattice, in Proceedings, 35th International Symposium on Lattice Field Theory (Lattice2017): Granada, Spain, to appear in EPJ Web Conf.,

[2] S. Borsanyi et al., Nature 539, 69 (2016), 1606.07494

[3] S. Borsanyi, Z. Fodor, C. Hoelbling, S.D. Katz, S. Krieg, C. Ratti, K.K. Szabo (WuppertalBudapest), JHEP 09, 073 (2010), 1005 . 3508 


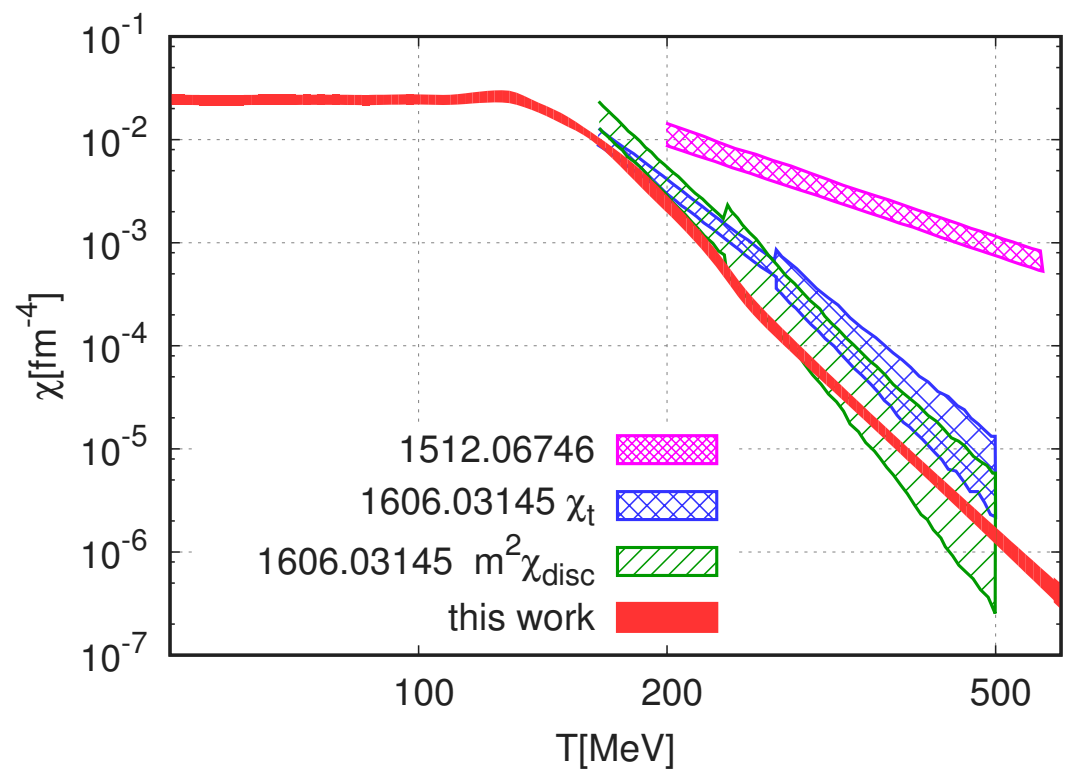

Figure 6. Our main result, the temperature-dependence of the QCD topological susceptibility. For comparison we also included two other results from the literature. 1512.06746 refers to Bonati et al. $[9,13]$ and 1606.03145 to Petreczky et al. [14]. In each case the bands indicate the uncertainty quoted by the authors.

[4] J. Frison, R. Kitano, H. Matsufuru, S. Mori, N. Yamada, JHEP 09, 021 (2016), 1606.07175

[5] C. Morningstar, M.J. Peardon, Phys. Rev. D69, 054501 (2004), hep-lat/0311018

[6] H. Neuberger, Phys. Lett. B417, 141 (1998), hep-lat/9707022

[7] S. Borsanyi, Z. Fodor, S.D. Katz, S.F. Krieg, T. Lippert, D. Nogradi, F. Pittler, K.K. Szabo, B.C. Toth (2015), 1510.03376

[8] S. Borsanyi, Y. Delgado, S. Durr, Z. Fodor, S.D. Katz, S. Krieg, T. Lippert, D. Nogradi, K.K. Szabo, Phys. Lett. B713, 342 (2012), 1204.4089

[9] C. Bonati, M. D’Elia, M. Mariti, G. Martinelli, M. Mesiti, F. Negro, F. Sanfilippo, G. Villadoro, JHEP 03, 155 (2016), 1512 . 06746

[10] A. Hasenfratz, C. Nieter, Phys. Lett. B439, 366 (1998), hep-lat/9806026

[11] M.F. Atiyah, I.M. Singer, Annals Math. 87, 484 (1968)

[12] M. Lüscher, JHEP 08, 071 (2010), [Erratum: JHEP03,092(2014)], 1006. 4518

[13] C. Bonati, The topological properties of QCD at high temperature: problems and perspectives, in Proceedings, 35th International Symposium on Lattice Field Theory (Lattice2017): Granada, Spain, to appear in EPJ Web Conf.,

[14] P. Petreczky, H.P. Schadler, S. Sharma, Phys. Lett. B762, 498 (2016), 1606.03145 